\title{
INVARIANT TRACE-FIELDS AND QUATERNION ALGEBRAS OF POLYHEDRAL GROUPS
}

\author{
C. MACLACHLAN AND A. W. REID
}

\section{Introduction}

Let $P$ be a polyhedron in $\mathbf{H}^{3}$ of finite volume such that the group $\Gamma(P)$ generated by reflections in the faces of $P$ is a discrete subgroup of Isom $\mathbf{H}^{3}$. Let $\Gamma^{+}(P)$ denote the subgroup of index 2 consisting entirely of orientation-preserving isometries so that $\Gamma^{+}(P)$ is a Kleinian group of finite covolume. $\Gamma^{+}(P)$ is called a polyhedral group.

As discussed in [12] and [13] for example (see \$2 below), associated to a Kleinian group $\Gamma$ of finite covolume is a pair $(A \Gamma, k \Gamma)$ which is an invariant of the commensurability class of $\Gamma ; k \Gamma$ is a number field called the invariant trace-field, and $A \Gamma$ is a quaternion algebra over $k \Gamma$. It has been of some interest recently $(\mathrm{cf} .[\mathbf{1 3}, \mathbf{1 6}])$ to identify the invariant trace-field and quaternion algebra associated to a Kleinian group $\Gamma$ of finite covolume since these are closely related to the geometry and topology of $\mathbf{H}^{3} / \Gamma$.

In this paper we give a method for identifying these in the case of polyhedral groups avoiding trace calculations. This extends the work in [15] and [11] on arithmetic polyhedral groups. In $\S 6$ we compute the invariant trace-field and quaternion algebra of a family of polyhedral groups arising from certain triangular prisms, and in $\$ 7$ we give an application of this calculation to construct closed hyperbolic 3-manifolds with 'non-integral trace'.

\section{Invariant trace-field and quaternion algebra}

Let $\Gamma$ be a finitely generated non-elementary subgroup of $\operatorname{PSL}(2, \mathbf{C})$ and $\Gamma^{(2)}=$ $\left\langle\gamma^{2} \mid \gamma \in \Gamma\right\rangle$.

Define $\hat{\Gamma}=\mathrm{P}^{-1}(\Gamma)$ where $\mathrm{P}$ is the natural projection $\mathrm{P}: \operatorname{SL}(2, \mathbf{C}) \longrightarrow \operatorname{PSL}(2, \mathbf{C})$. The trace-field of $\Gamma$ is $\mathbf{Q}(\operatorname{tr} \Gamma)=\mathbf{Q}(\operatorname{tr} \hat{\gamma}: \hat{\gamma} \in \hat{\Gamma})$, and is a finite extension of $\mathbf{Q}$ in the case where $\Gamma$ has finite covolume ([18, Chapter 5] and [9]). The invariant trace-field $k \Gamma$ and quaternion algebra $A \Gamma$ are given by the following theorem, see $[1,13,16]$.

TheOREM 2.1. The field $\mathbf{Q}\left(\operatorname{tr} \Gamma^{(2)}\right):=k \Gamma$ is an invariant of the commensurability class of $\Gamma$.

The algebra

$$
A \Gamma=\left\{\sum a_{i} \hat{\gamma}_{i} \mid a_{i} \in k \Gamma, \gamma_{i} \in \Gamma^{(2)}\right\}
$$

(where all sums are finite) is a quaternion algebra over $k \Gamma$ which is an invariant of the commensurability class of $\Gamma$.

Received 25 March 1996.

1991 Mathematics Subject Classification 57M50.

J. London Math. Soc. (2) 58 (1998) 709-722 
We now relate this invariant trace-field to the minimal field of definition as discussed in [20]. For this we introduce some terminology, some of which is used in [20].

For a vector space $V$ over $\mathbf{C}$ and a subgroup $\Delta$ of $\mathrm{GL}(V), \Delta$ is definable over a field $K$ if there is a $K$-lattice of $V$ which is invariant under $\Delta$; that is if, with respect to a suitable basis of $V, \Delta$ can be represented by matrices with entries in $K$. We state Vinberg's results only for the case where the ambient group is $\mathrm{PGL}_{2}$. These results are concerned with a Zariski dense subgroup $\Gamma$ and we note that, if $\Gamma$ is a Kleinian group of finite covolume in $\operatorname{PGL}(2, \mathbf{C})$, then $\Gamma$ is Zariski dense by Borel's density theorem (for example, [21, p. 41]). Before extending the notion of a group being definable over $K$, we recall the following.

If $G$ is an algebraic subgroup of $\operatorname{GL}(n, \mathbf{C})$ and $J \subset \mathbf{C}$ is a subring, we denote by $G(J)$ the subgroup $G \cap \mathrm{GL}(n, J)$. If $K \subset \mathbf{C}$ is a field, we say an algebraic group $H$ is a $K$-form of $\mathrm{PGL}_{2}$ if $H$ is definable over $K$ and is isomorphic to $\mathrm{PGL}_{2}$ over some extension of $K$.

Definition 2.2. $K$ is a field of definition of $\Gamma$ if there is a $K$-form $H$ of $\mathrm{PGL}_{2}$ and an isomorphism $\rho: \mathrm{PGL}_{2} \longrightarrow H$ defined over some finite extension of $K$ such that $\rho(\Gamma) \subset H(K)$.

Recall (see [14] for instance) that every $K$-form of $\mathrm{PGL}_{2}$ is of the form

$$
\frac{A^{*}}{Z(A)^{*}} \cong \mathrm{SO}\left(A_{0}, n\right)
$$

where $A$ is a quaternion algebra over $K$ and the subspace $A_{0}$ of pure quaternions is a quadratic space with quadratic map $n$ being the restriction of the norm. In the case where $A=M_{2}$, then $A_{0}=\mathscr{L}=s \ell(2)$, the subspace of traceless matrices, and the above isomorphism is induced by $\mathrm{Ad}$, the adjoint representation of $\mathrm{SL}_{2}$ into $\operatorname{GL}(\mathscr{L})$.

THEOREM 2.3 (Vinberg). Let $\Gamma$ be a Zariski dense subgroup of $\mathrm{PGL}_{2}$.

(i) $\mathrm{Ad} \Gamma$ is definable over $K$ if and only if $\mathbf{Q}(\operatorname{tr} \operatorname{Ad} \Gamma) \subset K$.

(ii) If $\Gamma_{1} \subset \Gamma$ of finite index, the classes of fields over which $\Gamma_{1}$ and $\Gamma$ are defined are identical.

(iii) If $K$ is a field of definition of $\Gamma$, then $\operatorname{Ad} \Gamma$ is definable over $K$.

For $\gamma \in \Gamma$, and $\hat{\gamma}$ as above, let $\hat{\gamma}$ have distinct eigenvalues $\lambda, \lambda^{-1}$, so that $\hat{\gamma}$ can be represented by

$$
\left(\begin{array}{cc}
\lambda & 0 \\
0 & \lambda^{-1}
\end{array}\right)
$$

Then from Figure 1 it follows easily that $\operatorname{Ad}(\hat{\gamma})$ has eigenvalues $1, \lambda^{2}, \lambda^{-2}$. Now

$$
\operatorname{tr}(\hat{\gamma})^{2}=\operatorname{tr}^{2}(\hat{\gamma})-2=\left(\lambda+\lambda^{-1}\right)^{2}-2=\operatorname{tr} \operatorname{Ad}(\hat{\gamma})-1 .
$$

The same formula holds if $\hat{\gamma}$ has equal eigenvalues and so $\gamma$ is parabolic.

THEOREM 2.4. Let $\Gamma$ be a finite covolume Kleinian group. Then $k \Gamma$ is the minimal field of definition of $\Gamma$ so that $K$ is a field of definition of $\Gamma$ if and only if $k \Gamma \subset K$. 


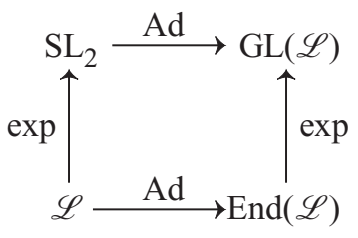

FIG. 1.

Proof. Note from above that $k \Gamma=\mathbf{Q}(\operatorname{tr} \operatorname{Ad} \Gamma)$. By Theorem 2.3(iii) and (i), it is immediate that if $K$ is a field of definition of $\Gamma$, then $k \Gamma \subset K$. Now each element of $\Gamma$ induces an automorphism of $\Gamma^{(2)}$ by conjugation and hence an automorphism of the invariant quaternion algebra $A \Gamma$. Since each automorphism of a quaternion algebra is inner, it follows that $\Gamma$ embeds in the $k \Gamma$ points of $A \Gamma^{*} / Z(A \Gamma)^{*}$. Thus $k \Gamma$ is a field of definition of $\Gamma$.

\section{Gram matrices}

Now let $P$ be a polyhedron as described in the introduction. By representing $P$ in the Lobachevski model of $\mathbf{H}^{3}$, we obtain the Gram matrix $G(P)$ as follows [19].

Consider $V \cong \mathbf{R}^{4}$ equipped with a non-degenerate inner product

$$
\langle\mathbf{x}, \mathbf{x}\rangle=-x_{0}^{2}+x_{1}^{2}+x_{2}^{2}+x_{3}^{2}
$$

and let $C^{+}=\left\{\mathbf{x} \mid\langle\mathbf{x}, \mathbf{x}\rangle\left\langle 0, x_{0}\right\rangle 0\right\}$. Then a model for $\mathbf{H}^{3}$ is $P C^{+}$and the isometries of $P C^{+}$are induced by the automorphisms of $(V,\langle\rangle$,$) which preserve the component$ $C^{+}$.

If a vector $\mathbf{v} \in V$ has positive norm, then its orthogonal complement is 3dimensional and is hyperbolic in the sense that the restriction of the inner product is non-degenerate and indefinite. Its projection to $\mathrm{PC}^{+}$is a hyperbolic plane.

A polyhedron $P$ in $\mathbf{H}^{3}$ will be the projection of a polyhedral angle $\mathscr{P}$ in $V$ bounded by linear hyperplanes through the origin. For each hyperplane choose an outward pointing normal $\mathbf{e}_{\mathbf{i}}$ and normalise such that $\left\langle\mathbf{e}_{\mathbf{i}}, \mathbf{e}_{\mathbf{i}}\right\rangle=1$. The polyhedral angle $\mathscr{P}$ is defined by $\left\langle\mathbf{e}_{\mathrm{i}}, \mathbf{x}\right\rangle \leqslant 0$. The Gram matrix $G(P)$ of this system of vectors is $G(P)=\left[a_{i j}\right]$ where $a_{i j}=\left\langle\mathbf{e}_{\mathbf{i}}, \mathbf{e}_{\mathbf{j}}\right\rangle$. If the dihedral angle between the planes $H_{i}, H_{j}$ is $\alpha$ then $a_{i j}=$ $-\cos \alpha$. More generally $a_{i j}=-\left(H_{i}, H_{j}\right)$ where $\left(H_{i}, H_{j}\right)$ is the inversive product of the hyperbolic planes in $\mathbf{H}^{3}$. If $P$ has $n$ faces $G(P)$ is an $n \times n$ matrix, but in all cases $G(P)$ has rank 4.

If $\Gamma$ is generated by reflections $\left\{r_{i}\right\}_{1 \leqslant i \leqslant n}$ in the faces of $P$, the reflection $r_{i}$ in the hyperplane $H_{i}$ is given by

$$
r_{i}(\mathbf{v})=\mathbf{v}-2\left\langle\mathbf{v}, \mathbf{e}_{\mathbf{i}}\right\rangle \mathbf{e}_{\mathbf{i}}
$$

and so $r_{i}$ can be represented by an $n \times n$ matrix $X$ such that $X^{t} G(P) X=G(P)$.

For any

$$
\left\{i_{1}, i_{2}, \ldots, i_{k}\right\} \subset\{1,2, \ldots, n\}
$$

define the cyclic products

$$
b_{i_{1} i_{2} \cdots i_{k}}=2^{k} a_{i_{1} i_{2}} a_{i_{2} i_{3}} \cdots a_{i_{k-1} i_{k}} a_{i_{k} i_{1}}
$$

and let $K(P)=\mathbf{Q}\left(\left\{b_{i_{1} i_{2} \cdots i_{k}}\right\}\right)$. It is not difficult to see that it suffices to assume in the definition of $K(P)$ that the suffices $\left\{i_{1}, i_{2}, \ldots, i_{k}\right\}$ are distinct. 
Consider the following vectors

$$
\mathbf{v}_{i_{1} i_{2} \cdots i_{k}}=2^{k} a_{1 i_{1}} a_{i_{1} i_{2}} \cdots a_{i_{k-1} i_{k}} \mathbf{e}_{i_{k}}
$$

with $\mathbf{v}_{1}=2 \mathbf{e}_{1}$ and the suffices defined as above.

The $K(P)$-module $M(P)$ spanned by $\left\{\mathbf{v}_{i_{1} i_{2} \cdots i_{k}}\right\}$ is a $\Gamma$ module. For if $r_{i}$ is as above,

We also have that

$$
\begin{aligned}
r_{i}\left(\mathbf{v}_{i_{1} i_{2} \cdots i_{k}}\right) & =\mathbf{v}_{i_{1} i_{2} \cdots i_{k}}-2\left\langle\mathbf{v}_{i_{1} i_{2} \cdots i_{k}}, \mathbf{e}_{\mathbf{i}}\right\rangle \mathbf{e}_{\mathbf{i}} \\
& =\mathbf{v}_{i_{1} i_{2} \cdots i_{k}}-2^{k+1} a_{1 i_{1}} \cdots a_{i_{k} i} \mathbf{e}_{\mathbf{i}} .
\end{aligned}
$$

$$
\begin{aligned}
\left\langle\mathbf{v}_{i_{1} i_{2} \cdots i_{k}}, \mathbf{v}_{j_{1} j_{2} \cdots j_{l}}\right\rangle & =2^{k} a_{1 i_{1}} a_{i_{1} i_{1}} \cdots a_{i_{k-1} i_{k}} 2^{l} a_{1 j_{1}} a_{j_{1} j_{2}} \cdots a_{j_{l-1} j_{l}} a_{i_{k} j_{l}} \\
& =1 / 2\left(2^{k+l+1} a_{1 i_{1}} \cdots a_{i_{k-1} i_{k}} a_{i_{k} j_{l}} a_{j_{l} j_{l-1}} \cdots a_{j_{1} 1}\right) \in K(P) .
\end{aligned}
$$

Thus if $\mathbf{u}, \mathbf{u}^{\prime} \in M(P)$ then $\left\langle\mathbf{u}, \mathbf{u}^{\prime}\right\rangle \in K(P)$. Now the real dimension of $M$ is clearly 4 and hence by the above its $K(P)$-dimension is also 4 .

With the restriction of the inner product, $M(P)$ is a quadratic space over $K(P)$ and so we have a representation of $\Gamma$ into the orthogonal group $O(M(P))$. Let the associated quadratic map be $q_{P}$ and pick an orthogonal basis $\mathbf{x}_{1}, \mathbf{x}_{2}, \mathbf{x}_{3}, \mathbf{x}_{4}$ of $M(P)$. The signed determinant of $M(P)$ is the element of $K(P)^{*} / K(P)^{* 2}$ represented by $d=$ $q_{P}\left(\mathbf{x}_{1}\right) q_{P}\left(\mathbf{x}_{2}\right) q_{P}\left(\mathbf{x}_{3}\right) q_{P}\left(\mathbf{x}_{4}\right)$.

We now state the main theorem. In this we let $k P$ and $A P$ denote the invariant trace-field and quaternion algebra of the group $\Gamma^{+}(P)$.

THeORem 3.1. (i) $[k P: k P \cap \mathbf{R}]=2$ and $K(P)=k P \cap \mathbf{R}$.

(ii) $k P=K(P)(\sqrt{d})$.

(iii) $A P \cong\left(\frac{-q_{P}\left(\mathbf{x}_{1}\right) q_{P}\left(\mathbf{x}_{2}\right),-q_{P}\left(\mathbf{x}_{1}\right)\left(q_{P}\left(\mathbf{x}_{3}\right)\right.}{k P}\right)$.

REMARK 3.2. This theorem is proved in [11] and [15] for the arithmetic tetrahedral groups.

REMARK 3.3. The proof of Theorem 3.1 appears in $\S 5$.

\section{Clifford algebras}

Part of our proof relies on results on the Clifford algebras of the quadratic space $M(P)$. We first recall some general information on Clifford algebras which can be found, for example, in [6].

Let $V$ be a non-degenerate quadratic space over a number field $k$ with quadratic map $q$ and associated bilinear form $B$. Let us denote its group of isometries by $O(V, q)$ and recall that $O(V, q)$ is generated by reflections $\tau_{\mathbf{u}}$, defined, for $\mathbf{u}$ an anisotropic vector in $V$, by

$$
\tau_{\mathbf{u}}(\mathbf{x})=\mathbf{x}-\frac{2 B(\mathbf{x}, \mathbf{u})}{q(\mathbf{u})} \mathbf{u} .
$$

The determinant of $\tau_{\mathbf{u}}$ is -1 and each element of $\operatorname{SO}(V, q)$ is a product of an even number of reflections.

The Clifford algebra $C(V)$ for $(V, q)$ is a $k$-algebra containing $V$ whose multiplication is compatible with $q$ in the sense that for all $\mathbf{x} \in V, \mathbf{x}^{2}=q(\mathbf{x}) .1$. The Clifford algebra is universal with this property so that for any other $k$-algebra $D$, 
containing $V$ and compatible with $q$, there is a unique $k$-algebra homomorphism $\phi: C(V) \longrightarrow D$ such that $\phi(\mathbf{x})=\mathbf{x}$ for all $\mathbf{x} \in V$. If $\operatorname{dim} V=n$ and $\mathbf{x}_{1}, \mathbf{x}_{2}, \ldots, \mathbf{x}_{\mathbf{n}}$ is an orthogonal basis of $V$, then $\left\{\mathbf{x}_{1}^{\mathbf{e}_{1}} \mathbf{X}_{2}^{\mathbf{e}_{2}} \ldots \mathbf{x}_{\mathbf{n}}^{\mathbf{e}_{\mathbf{n}}}: \mathbf{e}_{\mathbf{i}}=0\right.$ or 1$\}$ is a basis of $C(V)$ so that $\operatorname{dim}$ $C(V)=2^{n}$. Clearly $C(V)$ acquires a $\mathbf{Z}_{2}$-grading with even and odd parts $C_{0}(V), C_{1}(V)$ having dimensions $2^{n-1}$. The structure of $C(V)$ depends crucially on the parity of the dimension of $V$.

Note that

$$
q(\mathbf{x}+\mathbf{y})-q(\mathbf{x})-q(\mathbf{y})=(\mathbf{x}+\mathbf{y})^{2}-\mathbf{x}^{2}-\mathbf{y}^{2}=\mathbf{x y}+\mathbf{y x}=2 B(\mathbf{x}, \mathbf{y})
$$

for $\mathbf{x}, \mathbf{y} \in V$. Thus $\mathbf{x}, \mathbf{y} \in V$ are orthogonal if and only if $\mathbf{x y}=-\mathbf{y x}$ in $C(V)$. Note that, if $\mathbf{u} \in V$ is anisotropic, then it has an inverse $\mathbf{u} / q(\mathbf{u})$ in $C(V)$. Conversely, if $\mathbf{u} \in V$ has an inverse $\mathbf{v} \in C(V)$, then $q(\mathbf{u}) \mathbf{v}=\mathbf{u u v}=\mathbf{u}$ and so $q(\mathbf{u}) \neq 0$. Thus $\mathbf{u} \in V$ has an inverse in $C(V)$ if and only if $\mathbf{u}$ is anisotropic. Now suppose that $\mathbf{u}$ is anisotropic. Then

$$
\tau_{\mathbf{u}}(\mathbf{x})=\mathbf{x}-\frac{\mathbf{x u}+\mathbf{u x}}{\mathrm{q}(\mathbf{u})} \mathbf{u}=-\mathbf{u x u}^{-1} .
$$

Each isometry $\sigma \in O(V, q)$ admits a unique extension $\hat{\sigma}$ to a $k$-algebra automorphism of $C(V)$ by the universal property of $C(V)$. When $\sigma$ is a product of reflections, this extension can be expressed explicitly using (4.1). On basis vectors

$$
\hat{\sigma}\left(\mathbf{x}_{\mathbf{i}_{1}} \mathbf{x}_{\mathbf{i}_{2}} \ldots \mathbf{x}_{\mathbf{i}_{\mathrm{r}}}\right)=\sigma\left(\mathbf{x}_{\mathbf{i}_{1}}\right) \sigma\left(\mathbf{x}_{\mathbf{i}_{2}}\right) \ldots \sigma\left(\mathbf{x}_{\mathbf{i}_{\mathrm{r}}}\right)
$$

and $\hat{\sigma}$ preserves the grading, so by restriction determines an automorphism of $C_{0}(V)$. Let

$$
\phi: O(V, q) \longrightarrow \operatorname{Aut}(C(V))
$$

be defined by $\phi(\sigma)=\hat{\sigma}$.

Now the opposite algebra of $C(V)$ is also an algebra in which multiplication is compatible with the quadratic map $q$. Since the underlying set is the same, the universal property of $C(V)$ shows that there exists a unique algebra antiautomorphism $\varepsilon$ of $C(V)$ which fixes $V$. Note that $\varepsilon\left(\mathbf{u}_{1} \mathbf{u}_{2} \ldots \mathbf{u}_{\mathbf{r}}\right)=\mathbf{u}_{\mathbf{r}} \ldots \mathbf{u}_{1}, \varepsilon$ has order 2 and preserves the grading.

If $\operatorname{dim} V=n$, let $\mathbf{z}=\mathbf{x}_{1} \mathbf{x}_{2} \ldots \mathbf{x}_{\mathrm{n}}$. When $n$ is odd, $\mathbf{z} \in C_{1}(V)$ and lies in the centre of $C(V)$. When $n$ is even, $\mathbf{z} \in C_{0}(V)$ and $\mathbf{z}$ lies in the centre of $C_{0}(V)$. Note that $\mathbf{z}^{2}=$ $(-1)^{n(n-1) / 2} q\left(\mathbf{x}_{1}\right) \ldots q\left(\mathbf{x}_{\mathbf{n}}\right)=d$, which, modulo $k^{* 2}$, is the signed determinant of $(V, q)$.

The mapping

$$
\phi: \mathrm{SO}(V, q) \longrightarrow \operatorname{Aut}\left(C_{0}(V)\right)
$$

has kernel $\{ \pm I\} \cap \mathrm{SO}(V, q)$. For, each $\sigma \in \mathrm{SO}(V, q)$ is a product of an even number of reflections. Thus, for all $x \in C(V), \hat{\sigma}(x)=v x v^{-1}$ where $v$ is the product of an even number of vectors, so that $v \in C_{0}(V)$. Thus, if $\hat{\sigma}(x)=x$ for all $x \in C_{0}(V)$, then $v \in$ $Z\left(C_{0}(V)\right)$ which is spanned by $1, \mathbf{z}$. Now the anti-automorphism $\varepsilon$ fixes $\mathbf{z}$ and so restricted to $C_{0}(V)$ is the usual conjugation. Now if $v=\mathbf{v}_{1} \mathbf{v}_{2} \ldots \mathbf{v}_{2 \mathrm{~m}}$ then $n(v)=$ $n\left(\mathbf{v}_{1} \mathbf{v}_{2}\right) \ldots n\left(\mathbf{v}_{2 \mathbf{m}-1} \mathbf{v}_{2 \mathbf{m}}\right)$. Also $n\left(\mathbf{w}_{1} \mathbf{w}_{2}\right)=\left(\mathbf{w}_{1} \mathbf{w}_{2}\right) \varepsilon\left(\mathbf{w}_{1} \mathbf{w}_{2}\right)=\mathbf{w}_{1} \mathbf{w}_{2}{ }^{2} \mathbf{w}_{1}=q\left(\mathbf{w}_{1}\right) q\left(\mathbf{w}_{2}\right) \in k$. Thus $n(v) \in k$. Thus if $v=a \mathbf{z}+b$ then either $a=0$ or $b=0$. Hence either $\sigma=I$ or $\sigma=-I$.

When $V$ has dimension 4 , then $\mathbf{z}$ lies in the centre of $C_{0}(V)$ and $C_{0}(V)$ is a central simple algebra of dimension 4 over the field spanned by $1, \mathbf{z}$ over $k$. When $k$ is a subfield of the reals and $V$ has signature $(3,1)$ then $\mathbf{z}^{2}=d$ is negative so that the quaternion algebra $C_{0}(V)$ is defined over the non-real field $(k(\sqrt{d}))$. 
Now every automorphism of $C_{0}(V)$ is inner and so via $\phi$ above we have

$$
\phi: \mathrm{SO}(V, q) \longrightarrow \frac{C_{0}(V)^{*}}{Z\left(C_{0}(V)\right)^{*}} .
$$

\section{Proof of Theorem 3.1}

5.1.

Part (i) of Theorem 3.1 is well known and follows from a more general result.

Lemma 5.1. Let $G$ be a finite covolume Kleinian group normalised by an orientation-reversing involution. Then $[k G: k G \cap \mathbf{R}]=2$.

Proof. Let $\sigma$ be the orientation-reversing involution, which by conjugating if necessary we can assume is the extension of complex conjugation to $\mathbf{H}^{3}$. Pass to a subgroup $G_{0}$ of finite index in $G$ for which $\mathbf{Q}\left(\operatorname{tr} G_{0}\right)$ coincides with $k G$ and $G_{0}$ is normalised by $\sigma$. Since $G_{0}$ is normalised by $\sigma$ it follows that if $g \in G_{0}$, then

$$
\overline{\operatorname{tr} g} \in \mathbf{Q}\left(\operatorname{tr} G_{0}\right)=k G .
$$

Since $G_{0}$ has finite covolume there are elements of non-real trace and it follows that complex conjugation preserves the non-real field $k G$. Therefore $[k G: k G \cap \mathbf{R}]=2$.

5.2.

The next thing we shall show is that $k P \cap \mathbf{R} \subset K(P)$. To do this we apply the results of $\S 4$ to the quadratic space $M(P)$ which is defined over the real field $K(P)$ and has signature $(3,1)$, with negative signed determinant $d$.

Now $\Gamma^{+}(P)$ embeds in $\mathrm{SO}(M(P))$. Since $\Gamma^{+}(P)$ has trivial centre, it follows from (4.3) that it embeds, via $\phi$, into

$$
\frac{C_{0}(M(P))^{*}}{Z\left(C_{0}(M(P))\right)^{*}}
$$

which by the results of $\S 4$ is a $K(P)(\sqrt{d})$-form. Thus $K(P)(\sqrt{d})$ is a field of definition of $\Gamma^{+}(P)$. Thus by Theorem 2.4, $k P \subset K(P)(\sqrt{d})$. Hence $k P \cap \mathbf{R} \subset K(P)(\sqrt{d}) \cap \mathbf{R}$ and $K(P)(\sqrt{d}) \cap \mathbf{R}=K(P)$ as required.

\section{3.}

For the reverse inclusion, we first note additional results proved in [20] and continue the notation of $\$ 2$. Consider the 4-dimensional space $V=\mathbf{R}^{4}$ equipped with the inner product of signature $(3,1)$ as before, so that we have an embedding $\mu$ of $\Gamma(P)$ into $O(V)$. As this leaves the $K(P)$-lattice $M(P)$ invariant, then $\mu(\Gamma(P))$ is definable over $K(P)$.

THEOREM 5.2 (Vinberg [20]). Let $\Gamma$ be Zariski dense in $O(V)$ and generated by reflections with associated Gram matrix $G$. Let $\Delta$ be any subgroup of finite index in $\Gamma$. Then $\Delta$ is definable over a field $K$ if and only if $K$ contains the cyclic products $b_{i_{1} i_{2} \cdots i_{k}}$ of $G$. 
Now let us consider again $\Gamma^{+}(P) \subset \operatorname{PSL}(2, \mathbf{C})$. Let $\sigma$ be a reflection in one of the faces of $P$ so that the centraliser $C(\sigma)$ of $\sigma$ in $\Gamma^{+}(P)$ will stabilise the hyperbolic plane $H$ which is spanned by the face of $P$. $C(\sigma)$ has finite covolume acting on $H$ but may contain reflections. However, it contains a non-elementary Fuchsian subgroup $F$ of index 2 and choosing a pair of non-commuting elements in $F^{(2)}$ as generators for the quaternion algebra $A P$ over $k P$ (see [10]), we have that

$$
A P \cong\left(\frac{a, b}{k P}\right)
$$

with $a, b \in \ell:=k P \cap \mathbf{R}$. By Lemma 5.1 we can take a basis $\{1, \alpha\}$ of $k P$ over $\ell$ with $\alpha^{2} \in \ell$ and $\alpha^{2}<0$.

Choose a standard basis $\{1, i, j, i j\}$ of $A P$ and define the conjugate-linear involution on $A P$ by

$$
\tau\left(a_{0}+a_{1} i+a_{2} j+a_{3} i j\right)=\overline{a_{0}}-\overline{a_{1}} i-\overline{a_{2}} j-\overline{a_{3}} i j .
$$

Then exactly as in [11] we have the following properties:

(1) $\tau(c x+d y)=\bar{c} \tau(x)+\bar{d} \tau(y)$ for $c, d \in k P$ and $x, y \in A P$.

(2) $\tau^{2}=1$

(3) $\tau(x y)=\tau(y) \tau(x)$ for $x, y \in A P$.

Furthermore if $V_{\tau}=\{x \in A P \mid \tau(x)=x\}$, then $V_{\tau}$ is a 4-dimensional $\ell$-vector with basis $\{1, \alpha i, \alpha j, \alpha i j\}$ and the norm form of $A P$ restricted to $V_{\tau}$ yields a quadratic form $q$ which with respect to the above basis is given by $\operatorname{diag}\left\{1,-\alpha^{2} a,-\alpha^{2} b, \alpha^{2} a b\right\}$. Thus over $\mathbf{R}, O\left(V_{\tau}\right) \cong O(V)$.

Now for each $y \in A P^{1}$, the group of elements of norm one in $A P$, define $\phi_{y}$ on $V_{\tau}$ by $\phi_{y}(x)=y x \tau(y)$. Using the properties of $\tau$ listed above we deduce the following.

Lemma 5.3. $\phi_{y}(x) \in V_{\tau}$ and $q\left(\phi_{y}(x)\right)=q(x)$.

Lemma 5.3 determines a homomorphism

$$
\Phi: A P^{1} \longrightarrow O\left(V_{\tau}, q ; \ell\right) .
$$

Following this by the isomorphism to $O(V)$, we obtain an embedding of $\Gamma^{+(2)}$ as a finite covolume subgroup. By Mostow rigidity, this embedding will be conjugate to $\mu$. Thus this subgroup of finite index is defined over $\ell$ and so by Theorem 5.2, $K(P) \subset \ell$.

5.4 .

We can now complete the proof of Theorem 3.1. In $\$ 5.2$ it was shown that $\ell \subset$ $K(P)$. $\$ 5.3$ proves the reverse inclusion so that this together with Lemma 5.1 proves Theorem 3.1(i). Since both indices $[K(P)(\sqrt{d}): K(P)]$, and $[k P: k P \cap \mathbf{R}]$ are 2, Theorem 3.1(ii) follows since $k P \subset K(P)(\sqrt{d})$. Finally, $\left\{1, \mathbf{x}_{1} \mathbf{x}_{2}, \mathbf{x}_{1} \mathbf{x}_{3},-q_{P}\left(\mathbf{x}_{1}\right) \mathbf{x}_{2} \mathbf{x}_{3}\right\}$ is a standard basis of $C_{0}(M(P))$, and Theorem 3.1(iii) follows from the remarks above and the proof of Theorem 2.4 .

We remark that the same method of proof can be used to construct the invariant trace-field and quaternion algebra of any finite covolume Kleinian group with a nonelementary Fuchsian subgroup, and which is normalised by an orientation-reversing involution. 


\section{Examples}

For $q \geqslant 7$, let $\Gamma_{q}$ denote the group generated by reflections in the triangular prism $P_{q}$ shown in Figure 2, and $\Gamma_{q}^{+}$denote the subgroup of index 2 (as in §2) (cf. [3]).

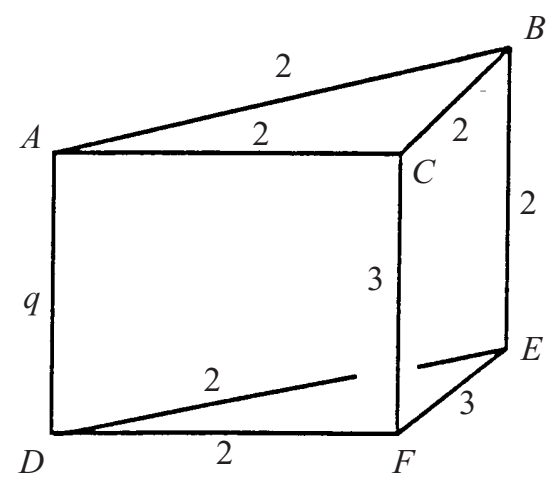

FIG. 2.

By [19] (see also [15]) the group $\Gamma_{q}^{+}$is arithmetic exactly when $q=7,8,9,10,14$. Using Theorem 3.1 and the method of proof we give the invariant trace-field and quaternion algebras for all $\Gamma_{q}^{+}$.

With the faces of the prism numbered as above we obtain the Gram matrix

$$
G_{q}=\left(\begin{array}{ccccc}
1 & -c & 0 & 0 & 0 \\
-c & 1 & -1 / 2 & 0 & 0 \\
0 & -1 / 2 & 1 & -1 / 2 & 0 \\
0 & 0 & -1 / 2 & 1 & -\cos \pi / q \\
0 & 0 & 0 & -\cos \pi / q & 1
\end{array}\right)
$$

where $c$ will be the inversive product defined by the two triangular faces. Since the rank of $G_{q}$ must be 4 , we can determine the value of $c$ as

$$
c^{2}=\frac{3 \cos ^{2} \pi / q-2}{4 \cos ^{2} \pi / q-3} .
$$

TheOREM 6.1. $k \Gamma_{q}^{+}=\mathbf{Q}(\sqrt{(2+2 \cos 2 \pi / q)(2-6 \cos 2 \pi / q)})$ and

$$
A \Gamma_{q}^{+} \cong\left(\frac{-1,-1}{k \Gamma_{q}^{+}}\right) \text {. }
$$

Proof. We proceed as above and compute an orthogonal basis for the quadratic space $M(P)$ over the field $K(P)$. It is not difficult to show that $K(P)=\mathbf{Q}(\cos 2 \pi / q)$. With the notation of $\S 2$, we choose $\mathbf{v}_{1}, \mathbf{v}_{2}, \mathbf{v}_{23}, \mathbf{v}_{234}$ so that $\mathbf{v}_{1}=\mathbf{2} \mathbf{e}_{1}, \mathbf{v}_{2}=-2 c \mathbf{e}_{2} \cdot \mathbf{v}_{23}=$ $2 c \mathbf{e}_{3}, \mathbf{v}_{234}=-2 c \mathbf{e}_{4}$. Then

$$
B_{q}=\left(\begin{array}{cccc}
4 & 4 c^{2} & 0 & 0 \\
4 c^{2} & 4 c^{2} & 2 c^{2} & 0 \\
0 & 2 c^{2} & 4 c^{2} & 2 c^{2} \\
0 & 0 & 2 c^{2} & 4 c^{2}
\end{array}\right)
$$


Diagonalising gives a form equivalent over $K\left(P_{q}\right)$ to

$$
\operatorname{diag}\left\{1,4 c^{2}, 3 c^{2}, 4 c^{2}\left(2-3 c^{2}\right) / 3\right\} \text {. }
$$

Thus the signed determinant $d=16 c^{6}\left(2-3 c^{2}\right)$ so that the invariant trace field is $\mathbf{Q}(\sqrt{(2+2 \cos 2 \pi / q)(2-6 \cos 2 \pi / q)})$. From the diagram of the prism given above, we see that the group $\Gamma_{q}^{+}$contains a subgroup isomorphic to $A_{4}$. Thus the invariant quaternion algebra is given by [5, Lemma 9.1]:

$$
\left(\frac{-1,-1}{\mathbf{Q}}\right) \otimes_{\mathbf{Q}} k \Gamma_{q}^{+} \cong\left(\frac{-1,-1}{k \Gamma_{q}^{+}}\right) .
$$

\section{Application}

Let $\Gamma$ be a subgroup of $\operatorname{SL}(2, \mathbf{C})$ (or $\operatorname{PSL}(2, \mathbf{C})$ ) whose traces consist of algebraic numbers. We say $\Gamma$ has non-integral trace if $\Gamma$ is not conjugate into $(\operatorname{SL}(2, \mathbf{A})$ (respectively $\operatorname{PSL}(2, \mathbf{A})$ ) where $\mathbf{A}$ is the ring of all algebraic integers in C. A remarkable theorem of Bass is the following which forms part of the $\mathrm{GL}_{2}$-subgroup theorem $[\mathbf{1}, \mathbf{2}]$.

THEOREM 7.1. Let $\Gamma$ be a finitely generated subgroup of $\operatorname{SL}(2, \mathbf{C})$ with a nonintegral trace. Then $\Gamma$ admits a splitting as a graph of groups in the sense of [17].

In the context of Kleinian groups and 3-manifolds, we have the following topological interpretation.

COROLlary 7.2. Let $M=\mathbf{H}^{3} / \Gamma$ be a closed hyperbolic 3-manifold such that $\Gamma$ has a non-integral trace. Then $M$ is Haken.

Very few examples of closed hyperbolic 3-manifolds with non-integral trace are known. Examples of link complements in $S^{3}$ are implicit in [13, §5-8]. Using the examples in $\S 6$, many examples will be obtained as a consequence of the calculations which follow.

Referring to Figure 2, we note that the edge of the prism which is the axis of an elliptic element of order $q$ is orthogonal to both triangular faces of the prism. Thus it is the unique common perpendicular to these two faces. Thus if $\lambda$ is the length of that edge, then $c=\cosh \lambda$ since $c$ is the inversive product of these two faces.

Consider a face of the prism which is a quadrilateral and contains this common perpendicular. Let $y_{1}, y_{2}$ denote the rotations of order 2 around the edges of this quadrilateral which abut the triangles. Then $y=y_{1} y_{2} \in \Gamma_{q}^{+}$and has the geodesic containing the perpendicular as its invariant axis. Note that $y_{1} y_{2}$ also leaves the plane spanned by the quadrilateral face invariant. Thus $y$ is a hyperbolic element with trace $2 c$. Thus $2 c$ is a trace in the group $\Gamma_{q}^{+}$. Thus $\operatorname{tr} y$ is an integer if and only if $4 c^{2}$ is an integer. Now

$$
4 c^{2}=3+\frac{1}{2 \cos 2 \pi / q-1}
$$

and so $4 c^{2}$ is an integer precisely when $2 \cos 2 \pi / q-1$ is a unit. 
7.1.

First, we want to determine when $2 \cos 2 \pi / q-1$ is a unit. Later we also need to know the norm of other linear combinations of $2 \cos 2 \pi / q$. This can be carried out as follows. Let $\Phi_{q}(x)$ denote the cyclotomic polynomial of degree $\phi(q)$, so that

$$
\Phi_{q}(x)=\prod_{(r, q)=1,1 \leqslant r<q / 2}\left(x^{2}-2 \cos \frac{2 r \pi}{q} x+1\right) .
$$

Thus for suitable choices of $x$ we can evaluate the norm of linear combinations of $2 \cos 2 \pi / q$ by evaluating the cyclotomic polynomial. In particular, for $x=\xi=\mathrm{e}^{\pi \mathrm{i} / 3}$, $x$ satisfies $x^{2}+1=x$ and so

$$
|N(2 \cos 2 \pi / q-1)|=\left|\Phi_{q}(\xi)\right| .
$$

Lemma 7.3. For $q \geqslant 7, u=2 \cos 2 \pi / q-1$ is a unit for all $q$ except

(i) $q=6 p^{\gamma}$ where $p$ is a prime $\neq 2,3$ and $\gamma \geqslant 1$, in which case $|N(u)|=p$;

(ii) $q=2^{\alpha} \cdot 3$ where $\alpha \geqslant 2$, in which case $|N(u)|=2$;

(iii) $q=2 \cdot 3^{\beta}$ where $\beta \geqslant 2$ in which case $|N(u)|=3$.

Proof. Let $q=d m$ where $m \neq 1$. Then

$$
\Phi_{q}(x) \mid \frac{x^{m d}-1}{x^{d}-1}=x^{d(m-1)}+x^{d(m-2)}+\ldots+x^{d}+1 .
$$

For $\eta=\xi^{ \pm 1}$, one has

$$
1+\eta+\ldots+\eta^{m-1}= \begin{cases}0 & \text { if } m \equiv 0(\bmod 6) \\ \text { a unit } & \text { if } m \equiv \pm 1(\bmod 6) \\ \sqrt{3} \times \text { a unit } & \text { if } m \equiv \pm 2(\bmod 6) \\ 2 \times \text { a unit } & \text { if } m \equiv 3(\bmod 6)\end{cases}
$$

Also, for $\eta=\xi^{ \pm 2}$, one has

$$
1+\eta+\ldots+\eta^{m-1}= \begin{cases}0 & \text { if } m \equiv 0(\bmod 3) \\ \text { a unit } & \text { if } m \equiv \pm 1(\bmod 3)\end{cases}
$$

and for $\eta=\xi^{3}$, one has

$$
1+\eta+\ldots+\eta^{m-1}= \begin{cases}0 & \text { if } m \equiv 0(\bmod 2) \\ 1 & \text { if } m \equiv 1(\bmod 2) .\end{cases}
$$

Thus let us assume that $q=2^{\alpha} 3^{\beta} m_{1} m_{2}$ where $\alpha, \beta \geqslant 0,\left(m_{1}, m_{2}\right)=1$ and $\left(m_{1} m_{2}, 6\right)=1$.

(1) If $\alpha=\beta=0$, take $d=1$ in (7.2) to obtain that $\Phi_{q}(\xi)$ is a unit. 
(2) If $\alpha=0, \beta>0$, take $d=3$ to obtain that $\Phi_{q}(\xi)$ is a unit.

(3) If $\alpha>0, \beta=0$, take $d=2$ to obtain that $\Phi_{q}(\xi)$ is a unit.

(4) If $\alpha, \beta>0$, taking $m=m_{2}$ in (7.2) gives that $\Phi_{q}(\xi) \mid m_{2}$ and then taking $m=m_{1}$ gives that $\Phi_{q}(\xi) \mid m_{1}$.

From these we deduce that $u$ is a unit unless $q=2^{\alpha} 3^{\beta} p^{\gamma}$ where $p$ is a prime $(\neq 2,3), \alpha, \beta>0$ and $\gamma \geqslant 0$.

(5) If $\alpha>1$, take $m=2$ so that $\Phi_{q}(\xi) \mid 2$.

(6) If $\beta>1$, take $m=3$ so that $\Phi_{q}(\xi) \mid 3$.

(7) If $\gamma \geqslant 1$, take $m=p$ so that $\Phi_{q}(\xi) \mid p$.

From these we see that $u$ is a unit unless $q=2^{\alpha} \cdot 3,2 \cdot 3^{\beta}, 6 p^{\gamma}$ where $\alpha, \beta \geqslant 2, \gamma \geqslant 1$. Now

$$
\Phi_{6 p^{\gamma}}(x)=\frac{\left(x^{3 p^{y}}+1\right)\left(x^{p^{\gamma-1}}+1\right)}{\left(x^{p^{y}}+1\right)\left(x^{3 p^{\gamma-1}}+1\right)}=\frac{x^{3 p^{y-1}(p-1)}-x^{3 p^{y-1}(p-2)}+\ldots+1}{x^{p^{y-1}(p-1)}-x^{p^{y-1}(p-2)}+\ldots+1} .
$$

Directly from this $\left|\Phi_{6 p^{p}}(\xi)\right|=p$. The other two cases are similar.

COROLlary 7.4. tr $y$ fails to be an integer precisely when $q=6 p^{\alpha}, 2^{a} 3,2 \cdot 3^{b}$.

7.2.

Here we extend the analysis of the non-integral traces further. First we state a result which is a strengthening of Theorem 7.1. The method of proof is that of [7], and we only sketch the relevant ideas. We need to recall the following. Let $k$ be a number field, and $\mathscr{P}$ be a $k$-prime divisor of the rational prime $p$. We say $\mathscr{P}$ is of degree 1 if the norm of $\mathscr{P}$ is $p$.

THEOREM 7.5. Let $\Gamma$ be a finitely generated subgroup of $\mathrm{SL}(2, k)$ with non-integral traces. Furthermore, assume there is a non-integral trace $t$ such that $\langle t\rangle=\mathscr{P}^{-a}$.I where $\mathscr{P}$ is a k-prime of degree 1 and $I$ is a fractional ideal for which $\mathscr{P}$ does not appear in its unique factorisation into prime ideals. Then $\Gamma$ splits as a non-trivial free product with amalgamation.

Sketch proof. Following the methods of [1] and [17], the degree 1 assumption on the prime $\mathscr{P}$ yields the existence of a faithful representation of $\Gamma$ into $\operatorname{SL}\left(2, \mathbf{Q}_{p}\right)$ where $\mathscr{P} \mid p$, and so we get an action of $\Gamma$ on the tree $T_{p}$ of $\mathrm{SL}_{2}$ over $\mathbf{Q}_{p}$. The non-integral trace assumption implies this action is non-trivial, and hence we deduce a splitting of $\Gamma$. The ideas of $[7, \S 5]$ show that $\Gamma$ is dense in $\operatorname{SL}\left(2, \mathbf{Q}_{p}\right)$ in the $p$-adic topology, and applying $[\mathbf{1 7}$, Theorem 3$]$ proves that the splitting is a free product with amalgamation.

Some of the groups $\Gamma_{q}^{+(2)}$ satisfy the hypothesis of Theorem 7.5 as we now show.

By Corollary 7.4 taking $q=6 p$ where $p$ is a prime greater than 5 gives that the groups $\Gamma_{q}^{+}$have non-integral trace. Note that the prime ideal $\mathscr{P}$ involved in the denominator is of degree 1 in $\mathbf{Q}(\cos 2 \pi / q)$. Let $\mathscr{P}=(2 \cos 2 \pi / q-1)$ in $\mathbf{Q}(\cos 2 \pi / q)$. 
We will now show that $\mathscr{P}$ splits in the extension $k \Gamma_{q}^{+} \mid \mathbf{Q}(\cos 2 \pi / q)$ for suitable choices of $p$. It will then follow from properties of primes in field extensions that the pair of primes in $k \Gamma_{q}^{+}$above such $p$ will be of degree 1 .

Recall from Theorem 6.1 that $k \Gamma_{q}^{+}$is generated by the element $\alpha=$ $\sqrt{(2+2 \cos 2 \pi / q)(2-6 \cos 2 \pi / q)}$ and we first show that $\mathscr{P}$ is unramified in the extension. The discriminant of the basis $1, \alpha$ of $k \Gamma_{q}^{+}$over $\mathbf{Q}(\cos 2 \pi / q)$ has norm $2^{\phi(q)} N(2+2 \cos 2 \pi / q) N(2-6 \cos 2 \pi / q)$. From (7.1) and (7.3), $N(2+2 \cos 2 \pi / q)=$ $\Phi_{q}(-1)=1$. Again from (7.1),

$$
N(2-6 \cos 2 \pi / q)=\left(\frac{3}{x_{0}}\right)^{\phi(q) / 2} \Phi_{q}\left(x_{0}\right)
$$

where $x_{0}=(1+2 \sqrt{-2}) / 3$. Now $\Phi_{q}(x) \mid x^{3 p}+1$ and so in the ring of integers in $\mathbf{Q}(\sqrt{-2})$, which is a Euclidean domain, $N(2-6 \cos 2 \pi / q)$, which is a rational integer, divides $(1+2 \sqrt{-2})^{3 p}+3^{3 p}$. Now

$$
(1+2 \sqrt{-2})^{3 p}+3^{3 p} \equiv\left[(1+2 \sqrt{-2})^{3}+3^{3}\right]^{p} \equiv[\sqrt{-2}(-1+\sqrt{-2})]^{3 p} \not \equiv 0(\bmod p) .
$$

Thus $\mathscr{P}$ is unramified in the extension $k \Gamma \mid \mathbf{Q}(\cos 2 \pi / q)$ and we can apply Kummer's theorem (for example, $[\mathbf{8}$, p. 20]). Reducing the minimum polynomial of $\alpha(\bmod \mathscr{P})$ gives $x^{2}+3$. Thus if we choose $p$ such that $(-3 / p)=1$ then $\mathscr{P}$ splits in the extension. These remarks give the following corollary.

COROllary 7.6. When $q=6 p$ where $p$ is a prime such that $p \equiv 1(\bmod 3)$, then the group $\Gamma_{q}^{+(2)}$ has a non-integral trace whose denominator lies in an ideal of $k \Gamma_{q}^{+}$of norm $p$.

By Dirichlet's theorem on primes in arithmetic progression, it follows that there are infinitely many such primes. Together with Theorem 7.5 we have the following corollary.

COROllary 7.7. With $q$ as in Corollary 7.6, let $\Gamma$ be a subgroup of finite index in $\Gamma_{q}^{+(2)}$. Then $\Gamma$ splits as a non-trivial free product with amalgamation.

The main interest to us here is not just the existence of this splitting but rather the passage from the group theoretical splitting to the existence of a closed incompressible surface. To prove our next theorem we will require some notation and to recall some standard facts; see [4, Chapter 1] for details.

Let $M$ be a compact orientable irreducible 3-manifold, $\tilde{M}$ denote the universal cover of $M$ and $p: \tilde{M} \longrightarrow M$ be the covering projection. Assume that $\pi_{1}(M)$ acts without inversions on a tree $T$. Let $E$ be the set of midpoints of edges in $T$.

A properly embedded surface $S$ in $M$ is associated to the action if there exists a $\pi_{1}(M)$-equivarient map $\tilde{\phi}: \tilde{M} \longrightarrow T$ which is transverse to $E$, and such that $\tilde{\phi}^{-1}(E)=$ $p^{-1}(S)$.

We have the following lemma (see [4, Proposition 1.3.6]).

Lemma 7.8. Let $S$ be a surface associated to the action of $\pi_{1}(M)$ on T. Suppose that $S^{\prime}$ is obtained from $S$ by compressing $S$ along a disc, then $S^{\prime}$ is also associated to the action. 
THEOREM 7.9. With $q$ as in Corollary 7.6 and $\Gamma$ a torsion-free subgroup of finite index in $\Gamma_{q}$ there is an incompressible component of the surface in $M=\mathbf{H}^{3} / \Gamma$ associated to the action of $\Gamma$ on $T_{p}$ (as above) which is non-separating.

Proof. With reference to Figure $2, \Gamma_{q}^{+}$can be generated by three elements $x, y$, $z$ where $x$ is the rotation around the edge $C F, y$ is the hyperbolic element described earlier, which is the product of the rotations around the edges $A C$ and $D F$ and $z$ is the rotation around $A D$. By suitably locating the prism in $H^{3}$, we obtain that $x, y, z$ are the images of the matrices

$$
X=\frac{1}{u}\left(\begin{array}{cc}
-1 & 2 \cos 2 \pi / q-1 \\
-\mathrm{e}^{2 \pi \mathrm{i} / q} & \mathrm{e}^{2 \pi \mathrm{i} / q}
\end{array}\right), \quad Y=\left(\begin{array}{cc}
t & 0 \\
0 & t^{-1}
\end{array}\right), \quad Z=\left(\begin{array}{cc}
\mathrm{e}^{\pi \mathrm{i} / q} & 0 \\
0 & \mathrm{e}^{-\pi \mathrm{i} / q}
\end{array}\right)
$$

where $u=\mathrm{e}^{2 \pi \mathrm{i} / q}-1$. Now the stabiliser in $\Gamma_{q}^{+}$of the plane $H$ spanned by the face $A B C$ is a $\mathbf{Z}_{2}$-extension of the triangle group $(2,3, q)$. However, on passing to $\Gamma_{q}^{+(2)}$ the corresponding stabiliser is the Fuchsian triangle group $F$ of signature $(3,3,3 p)$ which is generated by the images of $X$ and $Z^{2}$. We note that $X, Z^{2} \in \operatorname{SL}\left(2, \mathbf{Q}\left(\mathrm{e}^{2 \pi \mathrm{i} / q}\right)\right)$ and that the trace field $k F$ of $F$ is $\mathbf{Q}(\cos 2 \pi / q)$. Now $x, z^{2}$ generate a non-elementary subgroup of $\Gamma_{q}^{+(2)}$ so that the quaternion algebra $A F=k F\left[I, X, Z^{2}, X Z^{2}\right]$ and $A=A \Gamma_{q}^{+}=$ $k \Gamma\left[I, X, Z^{2}, X Z^{2}\right]$. Thus $A \Gamma \cong A F \otimes_{k F} k \Gamma$.

As before, let $\mathscr{P}$ be the ideal generated by $2 \cos 2 \pi / q-1$ in $k F$ and let $\mathscr{Q}$ be one of the ideals of $k \Gamma$ which divide $\mathscr{P}$. Since, by Theorem $6.1, A$ can only have finite ramification at dyadic primes

$$
\begin{aligned}
M_{2}\left((k \Gamma)_{2}\right) & \cong A \otimes_{k \Gamma}(k \Gamma)_{2} \cong A F \otimes_{k F} k \Gamma \otimes_{k \Gamma}(k \Gamma)_{\mathscr{Q}} \\
& \cong A F \otimes_{k F}(k F)_{\mathscr{P}} \cong M_{2}\left((k F)_{\mathscr{P}}\right) .
\end{aligned}
$$

Now $L=\mathbf{Q}\left(\mathrm{e}^{2 \pi \mathrm{i} / q}\right)$ splits the algebra $A F$ and, furthermore, the minimum polynomial of the generating element over $k F$ is

$$
x^{2}-2 \cos 2 \pi / q x+1 \equiv x^{2}-x+1(\bmod \mathscr{P}) .
$$

Since $(-3 / p)=1$, the prime $\mathscr{P}$ splits in the extension $L$. Let $\mathscr{R}$ be one of the primes in $L$ which divide $\mathscr{P}$ so that $L_{\mathscr{R}} \cong(k F)_{\mathscr{P}}$. In $M_{2}(L)$, since $N\left(\mathrm{e}^{2 \pi \mathrm{i} / q}-1\right)=\Phi_{q}(1)=1$, all entries of $X, Z^{2}$ are integral. Additionally, since $2 \cos 2 \pi / q-1$ can be chosen to be a uniformiser $\pi$ in $(k F)_{\mathscr{P}}$, then the images of $X, Z^{2}$ locally at 2 lie in the intersection of adjacent maximal orders of $A \Gamma \otimes_{k \Gamma}(k \Gamma)_{2}$ and so stabilise an edge of the tree $T_{p}$ [17].

Now if $\Gamma_{0}$ is any torsion-free subgroup of finite index in $\Gamma_{q}^{+(2)}$ we therefore deduce that an incompressible surface $S$ associated to the action given by Corollary 7.6 must contain a component that is totally geodesic since its fundamental group is a subgroup of $F$. Notice that the quotient $H / F$ gives rise to an embedded nonseparating 2-orbifold in the 3-orbifold $\mathbf{H}^{3} / \Gamma_{q}^{+(2)}$. Thus in the finite cover $\mathbf{H}^{3} / \Gamma_{0}$ we see an embedded totally geodesic non-separating surface. By construction this surface is associated to the action of $\Gamma_{0}$ on $T_{p}$ as above.

Acknowledgements. A. W. Reid is supported by the Royal Society. This author also wishes to thank the University of Aberdeen for its hospitality during the completion of this work. 


\section{References}

1. H. Bass, 'Groups of integral representation type', Pacific J. Math. 86 (1980) 15-51.

2. H. Bass, 'Finitely generated subgroups of $\mathrm{GL}_{2}$ ', The Smith conjecture (eds. J. W. Morgan and H. Bass; Academic Press, New York, 1984) 127-136.

3. M. D. E. Conder and G. J. Martin, 'Cusps, triangle groups and hyperbolic 3-folds', J. Austral. Math. Soc. 55 (1993) 149-182.

4. M. Culler, C. M. Gordon, J. Luecke and P. B. Shalen, 'Dehn surgery on knots', Ann. of Math. 125 (1987) 237-300.

5. F. W. Gehring, C. Maclachlan, G. J. Martin and A. W. Reid, 'Arithmeticity, discreteness and volume', Trans. Amer. Math. Soc. 349 (1987) 3611-3643.

6. T. Y. LAM, Algebraic theory of quadratic forms (Benjamin, New York, 1973)

7. D. D. Long, C. Maclachlan and A. W. ReID, 'Splitting groups of signature $(1 ; n)$ ', J. Algebra 185 (1996) 329-341.

8. R. L. Long, Algebraic number theory (Marcel Dekker, New York, 1977).

9. A. M. MaсbeAth, 'Commensurability of co-compact 3-dimensional hyperbolic groups', Duke Math. J. 50 (1983) 1245-1253.

10. C. Maclachlan and A. W. Reid, 'Commensurability classes of arithmetic Kleinian groups and their Fuchsian subgroups', Math. Proc. Cambridge Philos. Soc. 102 (1987) 251-257.

11. C. Maclachlan and A.W. ReID, 'The arithmetic structure of tetrahedral groups of hyperbolic isometries', Mathematika 36 (1989) 221-240.

12. W. Neumann and A. W. Reid, 'Amalgamation and the invariant trace field of a Kleinian group', Math. Proc. Cambridge Philos. Soc. 109 (1991) 509-515.

13. W. Neumann and A. W. ReID, 'Arithmetic of hyperbolic 3-manifolds', Topology '90, Proceedings of Low-Dimensional Topology Conference, Ohio State University (De Gruyter, New York, 1991) 273-310.

14. V. Platonov and A. RaPinchuk, Algebraic groups and number theory (Academic Press, New York, 1994).

15. A. W. REID, 'Arithmetic Kleinian groups and their Fuchsian subgroups', Ph.D. Thesis, Aberdeen University, 1987.

16. A. W. ReID, 'A note on trace-fields of Kleinian groups', Bull. London Math. Soc. 22 (1990) 349-352.

17. J.-P. SERRE, Trees (Springer, Berlin, 1980).

18. W. Thurston, 'The geometry and topology of 3-manifolds', lecture notes, Princeton, 1977.

19. E. B. VINBERG, 'Discrete groups in Lobachevskii space generated by reflections', Mat. Sb. 72 (1967) 471-488.

20. E. B. VINBERG, 'Rings of definition of dense subgroups of semisimple linear groups', Math. USSR Izvestija 5 (1971) 45-55.

21. R. J. ZIMMER, Ergodic theory and semisimple groups (Birkhäuser, 1984).

\section{Department of Mathematics University of Aberdeen \\ Aberdeen AB9 2TY}

E-mail: cmac@maths.abdn.ac.uk

\author{
Department of Mathematics \\ University of Texas \\ Austin \\ TX 78712 \\ USA
}

E-mail: areid@math.utexas.edu 